\title{
Wiener Chaos expansions and numerical solutions of randomly forced equations of fluid mechanics
}

\author{
Thomas Y. Hou *, Wuan Luo, Boris Rozovskii, Hao-Min Zhou \\ Department of Applied Mathematics, California Institute of Technology, 217-50, Pasadena, CA 91125, United States
}

Received 7 November 2004; received in revised form 11 November 2005; accepted 3 January 2006

Available online 17 February 2006

\begin{abstract}
In this paper, we propose a numerical method based on Wiener Chaos expansion and apply it to solve the stochastic Burgers and Navier-Stokes equations driven by Brownian motion. The main advantage of the Wiener Chaos approach is that it allows for the separation of random and deterministic effects in a rigorous and effective manner. The separation principle effectively reduces a stochastic equation to its associated propagator, a system of deterministic equations for the coefficients of the Wiener Chaos expansion. Simple formulas for statistical moments of the stochastic solution are presented. These formulas only involve the solutions of the propagator. We demonstrate that for short time solutions the numerical methods based on the Wiener Chaos expansion are more efficient and accurate than those based on the Monte Carlo simulations.
\end{abstract}

(c) 2006 Elsevier Inc. All rights reserved.

Keywords: Wiener Chaos expansions; Stochastic differential equations; Numerical methods; Navier-Stokes equations

\section{Introduction}

Stochastic partial differential equations (SPDEs) are known to be an effective tool in modeling complicated phenomena. Examples include wave propagation [25], diffusion through heterogeneous random media [26], randomly forced Burgers and Navier-Stokes equations (see e.g. $[2,5,15,23]$ and the references therein). Additional examples can be found in material science, chemistry, biology, and other areas. Many of the small scale effects and various uncertainties in these problems, which are difficult to deal with using traditional methods, can be naturally modeled by stochastic processes. Unlike deterministic partial differential equations, solutions of SPDEs are random fields. Hence, it is important to be able to deal with their statistical characteristics, e.g. mean, variance, and higher-order moments.

Due to the relatively complex nature of SPDEs, numerical simulations play an important role in the exploration of this important and useful class of PDEs. Currently, the Monte Carlo method is by far the most popular in simulating the effects modeled by SPDEs. The Monte Carlo method and its modifications, however,

\footnotetext{
${ }^{*}$ Corresponding author. Tel.: +1 626395 4546; fax: +1 6265780124.

E-mail address: hou@ama.caltech.edu (T.Y. Hou).
} 
have some limitations in applications to SPDEs with complex stochastic forcing, e.g., PDEs driven by Brownian motion. To correctly simulate small scale effects, one has to use a fine mesh to resolve the smallest possible scales. Moreover, many realizations have to be computed in order to obtain reliable estimates of various statistical properties. Therefore, Monte Carlo simulations are generally very expensive.

In this paper, we deal with Burgers and Navier-Stokes equation with Brownian-motion type forcing. These equations are important due to their role in modeling of certain turbulent effects (for detailed discussions, see $[1,15,17]$ and the references therein).

The method of attack is based on the Fourier analysis with respect to the Gaussian (rather than Lebesgue) measure. More specifically, the approach taken in this paper is based on the Cameron-Martin version of the Wiener Chaos expansion (see, e.g. $[3,12]$ and the references therein). Sometimes, the Wiener Chaos expansion (WCE for short) is also referred to as the Hermite polynomial chaos expansion.

The WCE of a solution of an SPDE separates the deterministic effects from the randomness. In particular, the Fourier coefficients of a white noise driven Navier-Stokes equation verify a system of coupled deterministic PDEs, which we refer to as the propagator (see, e.g. [22,23]). The propagator is a deterministic mechanism responsible for the evolution of randomness inherent to the original stochastic PDE. Remarkably, the propagator has the same type of nonlinearity as the original equation. Once the propagator is determined, standard deterministic numerical methods can be applied to solve it efficiently. The main statistics (such as mean, covariance, and higher-order statistical moments) can be calculated by simple formulas involving only the coefficients of the propagator. In the WCE approach, there is no randomness directly involved in the computations. One does not have to rely upon pseudo-random number generators, and there is no need to solve the stochastic PDEs repeatedly over many realizations. Instead, the propagator system is solved only once.

There is a long history of using WCE as well as other polynomial chaos expansions in problems in physics and engineering. See, e.g. $[9,24,6,7]$, etc. In particular, the papers $[9,24,6,7]$ deal with the contribution of loworder Wiener Chaos approximations to the inertial range spectrum of the Burgers equation. Ghanem and other authors have developed stochastic finite element methods based on the Karhunen-Loève expansion and Hermite polynomial chaos expansion [11,27]. Karniadakis et al. generalized this idea to other types of randomness and polynomials [13,30,31].

As mentioned above, this paper is mainly concerned with the equations of fluid dynamics driven by Brownian motion. Typically, applications of the polynomial chaos methodology to stochastic PDEs considered in the literature deal with stochastic input generated by a finite number of random variables (see, e.g. $[27,11,30,29])$. Usually, this assumption is introduced either directly or via a representation of the stochastic input by a truncated Karhunen-Loève expansion. In contrast, in our case it is necessary to deal constantly with a flux of new random variables (successive time increments of the driving Brownian motions). This effect complicates the problem drastically, even on a short time interval.

To mitigate this problem, we have introduced a compression technique that allows to reduce the number of Hermite polynomials in the expansion quite dramatically. The idea of the method is similar to the sparse tensor products developed by Schwab, etc. [10]. Also, to enhance the accuracy of the numerical simulations, we take advantage of the recently discovered analytical formulae for the coefficients of the WCE (see [23]). Previously, in the literature on numerical analysis of SPDEs, the coefficients were modeled numerically (Ghanem, personal communication).

The statistical characteristics of fluid flow forced by Brownian motion is far from being Gaussian (see, e.g. $[7,24]$ ). To address this problem, we have implemented a WCE based technique for computing moments of all orders. It is based on rigorous analytical formulae that involve only the deterministic coefficients of the WCE. In the past, numerical approximations based on polynomial chaos approach to equation of fluid dynamics dealt only with the first- and the second-order moments. In our simulations the statistical moments up to the fourth order were computed.

Practical application of the WCE requires a two way truncation: (a) with respect to the number $K$ of the random variables/wavelets and (b) with respect to the order $N$ of the Hermite polynomials involved. We have addressed the related error analysis problems both analytically and numerically. In particular, it was established that, at least in the case of spatially invariant forcing, the truncation error at time $\Delta t$ is given by the (asymptotic) formula 


$$
C\left[\left(\frac{\sigma^{2}(\Delta t)^{3}}{N+1}\right)^{\frac{N+1}{2}}+\left(\frac{\sigma^{2}(\Delta t)^{3}}{K^{3}}\right)^{1 / 2}\right]
$$

where $\sigma^{2}$ is the local variance of the Brownian forcing. This formula implies that the methods converges also globally at the rate $\mathrm{O}(\sqrt{\Delta t})$. To the best of our knowledge, no other analytical results on the WCE error analysis for the equations of fluid dynamics are available.

Numerical simulations are provided for stochastic Burgers equations with periodic boundary conditions and a 2D stochastic Navier-Stokes equation coupled with transport equation. In these experiments, we have compared the solution accuracy and the computational effectiveness of the WCE and the Monte Carlo (MC) approaches. In the case of spatial invariant forcing, we obtain a semi-analytic exact solution for the Burgers and the Navier-Stokes equations and use the semi-analytical solutions to verify the accuracy of the WCEbased method. Our numerical results demonstrate convincingly that the WCE-based method is more accurate than the corresponding MC computations. We also found that for short time computations, the numerical method based on the WCE is more efficient than the corresponding MC simulation at the same level of accuracy. The gain could be quite substantial in the one-dimensional case. The gain in the multi-dimensional case is offset to some extent by the rapid increase in the number of the WCE coefficients. However, the computational cost of the WCE method is still notably lower than the MC method, especially when the resolution level is high.

It is still very challenging to develop a WCE method which is effective for long time computations. In long time computations, the required number of WCE coefficients will increase rapidly which would make the direct applications of the WCE method much more difficult. We believe that the insight gained in the current study will be instrumental in the investigation of the long time WCE computations. In Section 6, we briefly discuss some modifications of the WCE that appears to be suitable for long time computations.

This paper is structured as follows: in Section 2, we outline the theoretical foundation of the Wiener Chaos expansion. In Section 3, by applying the WCE method to a stochastic Burgers equation, we illustrate the general procedure by deriving the Wiener Chaos propagator for a nonlinear SPDE. We also demonstrate the fast convergence and high accuracy of the WCE method for short time computations. In Section 4, we apply the WCE method to solving a stochastic Navier-Stokes equation modeling the roll-up of thin vorticity layers through the Kelvin-Helmholtz instability. In Section 5, a model error analysis of the WCE method is done in the case where the random forcing is independent of the spatial variables. In Section 6, we discuss some issues related to long time integrations of the WCE method. Brief conclusions are stated in Section 7.

\section{Wiener Chaos expansion}

Let $u$ be a solution of a generic SPDE

$$
u_{t}(x, t)=L(u)+\sigma \dot{W}(t)
$$

where $W(t)$ is a Brownian motion. A solution $u$ of this equation, is a function of $t, x$ and the Brownian motion path $W_{0}^{t}=\{W(s), 0 \leqslant s \leqslant t\}$. It would be beneficial if one could solve Eq. (2.1) by separating the spatialtemporal variables $(t, x)$ from the random variable $W_{0}^{t}$.

For any orthonormal basis $\left\{m_{i}(s), i=1,2, \ldots\right\}$ in $L^{2}([0, t])$, e.g., wavelets or trigonometric functions, define

$$
\xi_{i}=\int_{0}^{t} m_{i}(s) \mathrm{d} W(s), \quad i=1,2, \ldots
$$

It is easy to show that $\xi_{i}$ are independent identically distributed $\mathscr{N}(0,1)$ (standard Gaussian) random variables. It is a standard fact that

$$
W(s)=\int_{0}^{t} \chi_{[0, s]}(\tau) \mathrm{d} W(\tau)=\sum_{i=1}^{\infty} \xi_{i} \int_{0}^{s} m_{i}(\tau) \mathrm{d} \tau,
$$

where $\chi_{[0, s]}(\tau)$ is the characteristic function of interval $[0, s]$. The expansion (2.3) converges in the mean square sense 


$$
E\left[W(s)-\sum_{i=1}^{N} \xi_{i} \int_{0}^{s} m_{i}(\tau) \mathrm{d} \tau\right]^{2} \rightarrow 0 \quad \text { as } N \rightarrow \infty .
$$

for $s \leqslant t$ uniformly.

As a result, one can view $u(x, t)$ as a function of the spatial-temporal variable $(x, t)$ and random variables $\xi=\left(\xi_{1}, \xi_{2}, \ldots\right)$. These two groups of variables could be separated by a Fourier transform with respect to the tensor products of Hermite polynomials of $\xi$.

Denote the set of multi-indices with finite number of non-zero components as

$$
\mathscr{J}=\left\{\alpha=\left(\alpha_{i}, i \geqslant 1\right)\left|\alpha_{i} \in\{0,1,2 \ldots\},\right| \alpha \mid=\sum_{i=1}^{\infty} \alpha_{i}<\infty\right\}
$$

For an index $\alpha=\left(\alpha_{1}, \alpha_{2}, \ldots\right) \in \mathscr{J}$, a multi-variable Hermite polynomial of $\xi=\left(\xi_{1}, \xi_{2}, \ldots\right)$ is defined as

$$
T_{\alpha}(\xi)=\prod_{i=1}^{\infty} H_{\alpha_{i}}\left(\xi_{i}\right)
$$

where $H_{n}(x)$ is the normalized $n$ th-order Hermite polynomials [8]. For $\alpha \in \mathscr{J}$, the product in the right-hand side of (2.4) only has a finite number of factors and is well defined. The random functions $T_{\alpha}$ are often called Wick polynomials. They form a complete orthonormal basis in $L^{2}$ on the probability space with respect to the Gaussian measure generated by $\xi$. In particular, $E\left(T_{\alpha} T_{\beta}\right)=\delta_{\alpha \beta}$ where $E$ is the expectation symbol. Note that $E\left(T_{\alpha}\right)=0$ when $\alpha \neq \mathbf{0}$ and $E\left(T_{\mathbf{0}}\right)=T_{0}=1$. The order of the polynomial $T_{\alpha}$ is defined as $|\alpha|=\sum_{k} \alpha_{k}$. The following result, often referred to as the Cameron-Martin theorem, forms the foundation of Wiener Chaos theory.

Theorem 2.1 [3]. Assume that for fixed $x$ and $s \leqslant t, u(x, s)$ is a functional of the Brownian motion $W$ on the interval $[0, s]$ with $E|u(x, s)|^{2}<\infty$, then $u(x, s)$ has the following Fourier-Hermite expansion:

$$
u(x, s)=\sum_{\alpha \in \mathscr{I}} u_{\alpha}(x, s) T_{\alpha}, \quad u_{\alpha}(x, s)=E\left[u(x, s) T_{\alpha}\right],
$$

where $T_{\alpha}$ are the Wick polynomials defined by (2.4). Furthermore, the first two statistical moments of $u(x, s)$ are given by:

$$
E[u(x, s)]=u_{0}(x, s)
$$

and

$$
E\left[u^{2}(x, s)\right]=\sum_{\alpha \in \mathscr{J}}\left|u_{\alpha}(x, s)\right|^{2} .
$$

The Fourier-Hermite series (2.5) is often called the Wiener Chaos expansion (WCE) of $u(x, s)$.

In the following sections, we will develop the WCE for randomly forced Burgers and Navier-Stokes equations and apply it to compute the statistical moments of their solutions.

\section{WCE method for stochastic Burgers equations}

The stochastically forced Burgers equation has been an active research subject in recent years. Unlike its pure decaying unforced counterpart, the randomly forced Burgers equation has a much richer structure. It appears in a wide range of problems in statistical physics. As a test model, the randomly forced Burgers equation has also been studied in the context of turbulent flow. For detailed discussions, see $[1,15,16]$ and the references therein.

In this section, we consider the following 1D stochastic Burgers equation:

$$
\left\{\begin{array}{l}
u_{t}+\frac{1}{2}\left(u^{2}\right)_{x}=\mu u_{x x}+\sigma(x) \dot{W}(t), \\
u(x, 0)=u_{0}(x), \quad u(0, t)=u(1, t),
\end{array} \quad(t, x) \in(0, T] \times[0,1]\right.
$$


where $W(t)$ is a Brownian motion. For the sake of simplicity, we assume that the initial condition $u_{0}(x)$ is deterministic and limit our considerations to the periodic case. Other types of boundary conditions could be treated similarly. It is known (see, e.g. [4]) that if $\left\|u_{0}\right\|_{L_{2}}<\infty$ and $\|\sigma\|_{L_{2}}<\infty$, then Eq. (3.1) has a unique square integrable solution. Therefore, the solution of (3.1) admits a Wiener Chaos expansion. Below, we will derive a system of equations for the coefficients of the WCE and solve this system numerically. By comparing our results with those computed by Monte Carlo simulations and semi-analytic solutions, we will demonstrate the efficiency and the accuracy of the WCE method.

\subsection{Equations for WCE coefficients}

Let us fix an arbitrary orthonormal basis $\left\{m_{i}(t), i \geqslant 1\right\}$ in $L^{2}([0, T])$ and write $\xi_{i}=\int_{0}^{T} m_{i}(t) \mathrm{d} W(t)$. The Wick polynomials $T_{\alpha}$ associated with the Brownian process $W(t)$ are defined by (2.4). By the Cameron-Martin Theorem 2.1 the solution of the Burgers equation (3.1) admits the Wiener Chaos expansion (2.5). Furthermore, it can be shown that (see [23] for more details)

$$
u^{2}=\sum_{p \in \mathscr{J}} \sum_{0 \leqslant \beta \leqslant \alpha} C(\alpha, \beta, p) u_{\alpha-\beta+p} u_{\beta+p} T_{\alpha},
$$

where

$$
C(\alpha, \beta, p)=\left[\left(\begin{array}{c}
\alpha \\
\beta
\end{array}\right)\left(\begin{array}{c}
\beta+p \\
p
\end{array}\right)\left(\begin{array}{c}
\alpha-\beta+p \\
p
\end{array}\right)\right]^{\frac{1}{2}} .
$$

Moreover, we can deduce from formula (3.2) the following analytical formula to compute the third and the fourth-order moments:

$$
\begin{aligned}
& E\left[u^{3}\right]=E\left[u^{2} \cdot u\right]=\sum_{\alpha \in \mathscr{J}}\left(\sum_{p \in \mathscr{J}} \sum_{0 \leqslant \beta \leqslant \alpha} C(\alpha, \beta, p) u_{\alpha-\beta+p} u_{\beta+p}\right) u_{\alpha}, \\
& E\left[u^{4}\right]=E\left[u^{2} \cdot u^{2}\right]=\sum_{\alpha \in \mathscr{J}}\left(\sum_{p \in \mathscr{I}} \sum_{0 \leqslant \beta \leqslant \alpha} C(\alpha, \beta, p) u_{\alpha-\beta+p} u_{\beta+p}\right)^{2} .
\end{aligned}
$$

Using formula (3.2) and expansion (2.3), we can derive the governing equations for the WCE coefficients:

$$
\frac{\partial}{\partial t} u_{\alpha}(x, t)+\frac{1}{2} \sum_{p \in \mathcal{J}} \sum_{0 \leqslant \beta \leqslant \alpha} C(\alpha, \beta, p) \frac{\partial}{\partial x}\left(u_{\alpha-\beta+p} u_{\beta+p}\right)(x, t)=\mu \frac{\partial^{2}}{\partial x^{2}} u_{\alpha}(x, t)+\sigma(x) \sum_{i=0}^{\infty} I_{\left\{\alpha_{j}=\delta_{i j}\right\}} m_{i}(t),
$$

where for fixed $i, I_{\left\{\alpha_{j}=\delta_{i j}\right\}}$ equals 1 if $\alpha_{j}=\delta_{i j}$, and equals 0 otherwise. Since the initial condition $u_{0}(x)$ is deterministic, the initial conditions for the WCE coefficients are

$$
u_{\alpha}(x, 0)= \begin{cases}u_{0}(x), & \alpha=\mathbf{0} \\ 0, & \alpha \neq \mathbf{0} .\end{cases}
$$

Clearly, all the WCE coefficients still satisfy the periodic boundary condition $u_{\alpha}(0, t)=u_{\alpha}(1, t)$.

The WCE propagator (3.6) is a deterministic PDE system. The effect of the random forcing is captured by the $L^{2}$ basis functions $m_{i}(t)$. The first WCE coefficient $u_{\mathbf{0}}(x, t)$, which is the mean of the stochastic solution, satisfies

$$
\left(u_{\mathbf{0}}\right)_{t}+\frac{1}{2}\left(u_{\mathbf{0}}^{2}\right)_{x}+\frac{1}{2} \sum_{|\alpha| \neq 0}\left(u_{\alpha}^{2}\right)_{x}=\mu\left(u_{\mathbf{0}}\right)_{x x} .
$$

Clearly, the random forcing does not contribute to the mean $u_{\mathbf{0}}$ directly, since the forcing has zero mean. However, due to the nonlinearity, the mean is driven by higher-order WCE coefficients that represent the randomness of the solution. If we truncate the infinite system (3.6), then the truncated system can be viewed as a closure of the Reynolds equation of the mean. 


\subsection{Numerical solution}

In the following numerical experiment, we assume that the initial condition of the stochastic Burgers equation (3.1) is given by

$$
u_{0}(x)=\frac{1}{2}\left(\mathrm{e}^{\cos 2 \pi x}-1.5\right) \sin [2 \pi(x+0.37)] .
$$

We choose the spatial part of the random forcing as $\sigma(x)=\frac{1}{2} \cos (4 \pi x)$, and set the viscosity $\mu=0.005$.

The WCE propagator (3.6) is an infinite PDE system and has to be truncated for the numerical purpose. Recall that the WCE is a double infinite expansion:

$$
u(x, t)=\sum_{\alpha \in \mathscr{J}} u_{\alpha}(x, t) \prod_{i=1}^{\infty} H_{\alpha_{i}}\left(\xi_{i}\right) .
$$

Therefore, we need to truncate the WCE both ways. Suppose we want to keep only $K$ Gaussian random variables and Wick polynomials up to $N$ th-order in the WCE approximation. Define the truncated index set $\mathscr{J}_{K, N}=\left\{\alpha=\left(\alpha_{1}, \ldots, \alpha_{K}\right),|\alpha| \leqslant N\right\}$. Then the truncated WCE is given by

$$
u_{K, N}(x, t)=\sum_{|\alpha| \leqslant N} u_{\alpha}(x, t) \prod_{i=1}^{K} H_{\alpha_{i}}\left(\xi_{i}\right)=\sum_{\alpha \in \mathscr{Y}_{K, N}} u_{\alpha}(x, t) T_{\alpha} .
$$

The resulting approximation has altogether $\sum_{n=0}^{N}\left(\begin{array}{c}K+n-1 \\ n\end{array}\right)$ terms. The number of terms increases algebraically but very fast with respect to both $N$ and $K$. For a typical truncation $K=6$ and $N=4$, the finite WCE approximation has 210 coefficients. However, the simple truncation (3.10) is not optimal. The WCE decays both in the number of Gaussian variable $\xi_{i}$ and the Wick polynomial order. Note that for Wick polynomial $T_{\alpha}, \alpha=\left(\alpha_{1}, \alpha_{2}, \ldots, \alpha_{K}\right)$, the component $\alpha_{i}$ denotes the order of the Hermite polynomial for random variable $\xi_{i}$. Instead of using Hermite polynomials of the same order for all $\xi_{i}, i \leqslant K$, it is better to use lower-order polynomials for $\xi_{i}$ with higher subscripts. In addition to the truncation $\sum_{i=1}^{K} \alpha_{i} \leqslant N$, we introduce some extra constraints such as $\alpha_{i} \leqslant N-i$. This idea is similar to the sparse tensor product developed by Schwab, etc. (Theorem 4.9 of [10]) for solving random elliptic problems by polynomial chaos methods. For $K=6$ and $N=4$, we can use the maximum fourth-order Hermite polynomials for $\xi_{1}, \xi_{2}$, but only third-order Hermite polynomials for $\xi_{3}$, second-order Hermite polynomials for $\xi_{4}$ and first-order Hermite polynomials for $\xi_{5}, \xi_{6}$. We can also decouple the random variables $\xi_{5}, \xi_{6}$ from the rest of the random variables $\xi_{1}, \xi_{2}, \xi_{3}, \xi_{4}$. With the above compressions, the number of coefficients will be reduced dramatically, from 210 to 66 in the sparse truncation.

Next we solve the problem (3.1) with $T=0.8$ by the WCE method. By Theorem 5.1, for a spatially independent random forcing, the truncation error is proportional to $\sigma\left(\frac{T}{K}\right)^{3 / 2}$, where $\sigma$ is the magnitude of the random forcing, $T$ is the length of the time interval, and $K$ is the number of wavelets. For a given maximum mean square error tolerance $\epsilon$, we can approximately set

$$
K \simeq T\left(\frac{\sigma}{\epsilon}\right)^{2 / 3}
$$

In the current case, $T=0.8$ and $|\sigma| \leqslant 0.5$. Following the above guideline, we choose to project the Brownian motion $\{W(t), 0 \leqslant t \leqslant T\}$ onto the first eight bases of the Haar wavelets. The maximum mean square error for such a truncation is $\approx \mathrm{O}\left(10^{-2}\right)$. We truncate the WCE propagator to the first-order, second-order and thirdorder Wick polynomials, respectively. However, we only use first-order Hermite polynomials for Gaussian variables $\xi_{i}, i=5-8$ and decouple them from the Hermite polynomials of $\xi_{i}, i=1-4$. In the actual computations of the WCE approximations, only 9, 19 and 35 WCE coefficients are included, as compared with 9, 45 and $165 \mathrm{WCE}$ coefficients that are included in the full tensor products. The centered statistical moments computed from different orders of WCE approximations are compared in Fig. 1. The formula for the third-order moment and fourth-order moment are given by (3.4) and (3.5). The first-order WCE truncation is a piecewise Gaussian approximation. With only nine coefficients, the mean of the solution is captured quite well. That is because the Gaussian modes are dominant in magnitude and hence provide the leading order correction to the 

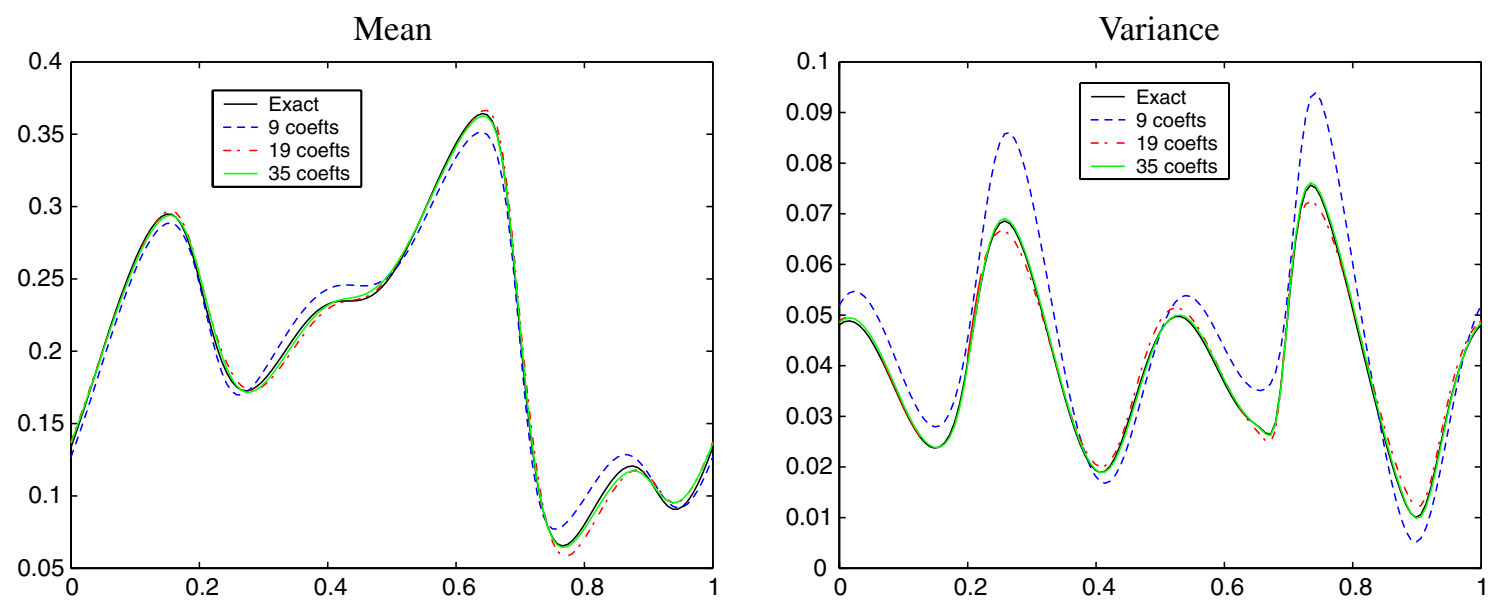

Third order moment
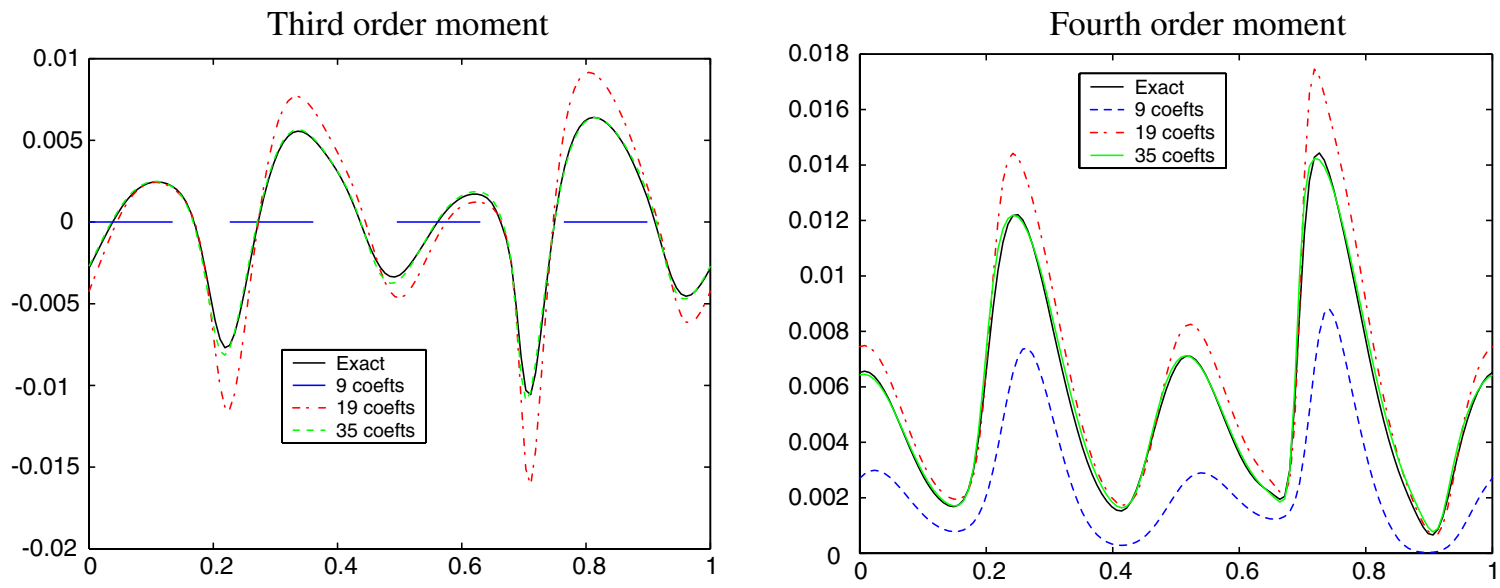

Fig. 1. Statistical moments computed by the WCE method with 9, 19 and 35 coefficients, respectively, corresponding to first-order, second-order, and third order WCE approximations. The exact solution is computed by MC simulation with 100,000 realizations. The first-order WCE approximation is a piecewise Gaussian approximation. It is obviously not accurate, especially for high-order moments. Including second-order corrections improves the results dramatically. The results given by the third order truncation are almost identical to the exact solutions.

mean Eq. (3.8). However, the stochastic solution of a nonlinear equation is by no means a Gaussian process. The first-order WCE approximation is inadequate for higher-order moments. Including second-order coefficients improves the numerical solutions dramatically, especially for the variance and the third-order moment. The numerical results of the third-order WCE approximation are very accurate and almost identical to the MC solutions with 100,000 realizations.

Fig. 2 is the $L_{2}$ norm of the WCE coefficients with third-order truncation. We order the multi-indices in the following way: if $|\alpha| \neq|\beta|$, then the index with smaller summation is listed first. Otherwise, the first component is compared. The index $\alpha$ will be listed ahead of $\beta$ if $\alpha_{1}>\beta_{1}$. If $\alpha_{1}=\beta_{1}$, then the second component is compared, and so on and so forth. In Fig. 2, the first coefficient is the mean, and coefficients $2-9$ correspond to the Gaussian part of the solution. The Gaussian coefficients decay quickly and the coefficients of $\xi_{8}$ is already very small. This partly confirms the truncation choice $K=8$ as predicted by Theorem 5.1 . The coefficients of $\xi_{k}, k=5-8$ are relatively small comparing with those of $\xi_{i}, i=1-4$, which justifies using only the first-order Hermite polynomials for $\xi_{k}, k=5-8$ and decoupling them from $\xi_{i}, i=1-4$. Coefficients 10-19 correspond to second-order Wick polynomials. They provide significant correction to the first-order approximation. Coefficients 20-35 correspond to third-order Wick polynomials. High-order coefficients are needed when resolving high-order moments. Fig. 2 shows that the WCE coefficients decay quickly as the order of 


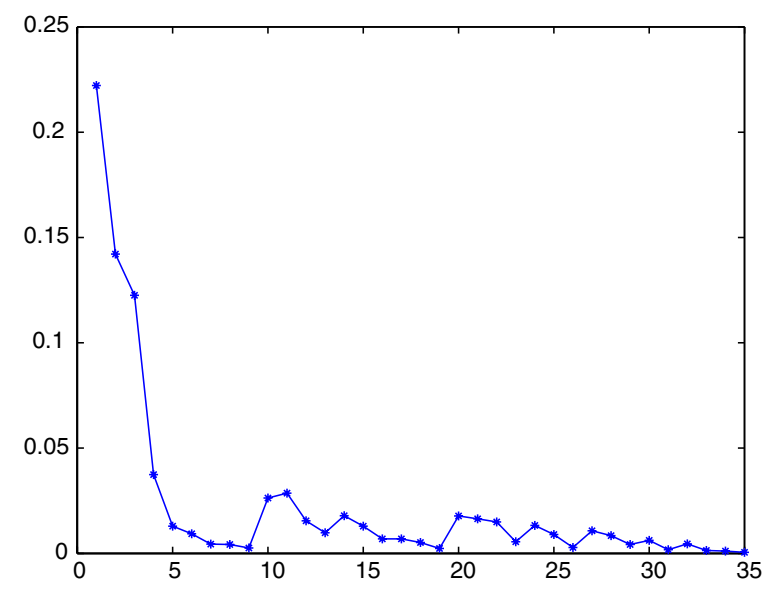

Fig. 2. $L^{2}$ norm of the WCE coefficients at third order truncation. The first 9 coefficients correspond to the Gaussian part of the solution; coefficients 10-19 correspond to second-order Wick polynomials; coefficients 20-35 correspond to third order Wick polynomials.

Wick polynomials increases. The rate of decay will depend on the variability and the length of the time interval, as indicated by our error analysis in Section 5. Larger variability will lead to slower decay in the WCE coefficients.

Fig. 3 is the qualitative comparison between the WCE method and MC simulation. The numerical results by the WCE method with 35 coefficients have similar accuracy as those by MC simulation with 10,000 realizations. However, the CPU time is only $2 \mathrm{~s}$ for the WCE method, as opposed to $315 \mathrm{~s}$ for MC simulation. Therefore, the WCE method is more efficient than MC simulation in this case. All the numerical experiments presented in this paper are conducted on a PC with a $2.60 \mathrm{GHz} \mathrm{CPU}$. In the MC simulation, we also experimented with various kind of acceleration techniques. However, most variance reduction technique are not readily applicable here since they require prior knowledge of the distribution of the stochastic solutions. Other acceleration techniques, such as antithetic variables, showed little advantage over the straightforward MC simulation when large enough number (order of thousands) of realizations are simulated.

\subsection{Convergence verification of the WCE method}

To demonstrate the convergence of the WCE method, we compare its numerical solutions with those of the MC simulation. However, it is difficult to obtain accurate solutions by MC simulation, due to its slow and non-monotone convergence. In this subsection, we will test both the WCE method and MC simulation on a model equation, where a semi-analytical solution is available. The semi-analytical solution can be computed very accurately by deterministic numerical algorithms. Using it as the benchmark, we will compare the performance of both the WCE method and MC simulation quantitatively.

In stochastic Burgers equation (3.1), suppose the spatial part $\sigma(x)$ of the random forcing is a constant. In this particular case the solution of the stochastic Burgers equation can be obtained by a nonlinear stochastic perturbation of its deterministic counterpart. More specifically, the following theorem holds:

Theorem 3.1. If $\sigma$ is constant, then the solution of (3.1) is given by

$$
u(x, t)=v\left(x-\sigma \int_{0}^{t} W(s) d s, t\right)+\sigma W(t),
$$

where $v(x, t)$ is a solution of the unforced deterministic Burgers equation

$$
\left\{\begin{array}{l}
v_{t}+\frac{1}{2}\left(v^{2}\right)_{x}=\mu v_{x x}, \\
v(x, 0)=u_{0}(x), v(0, t)=v(1, t) .
\end{array}\right.
$$



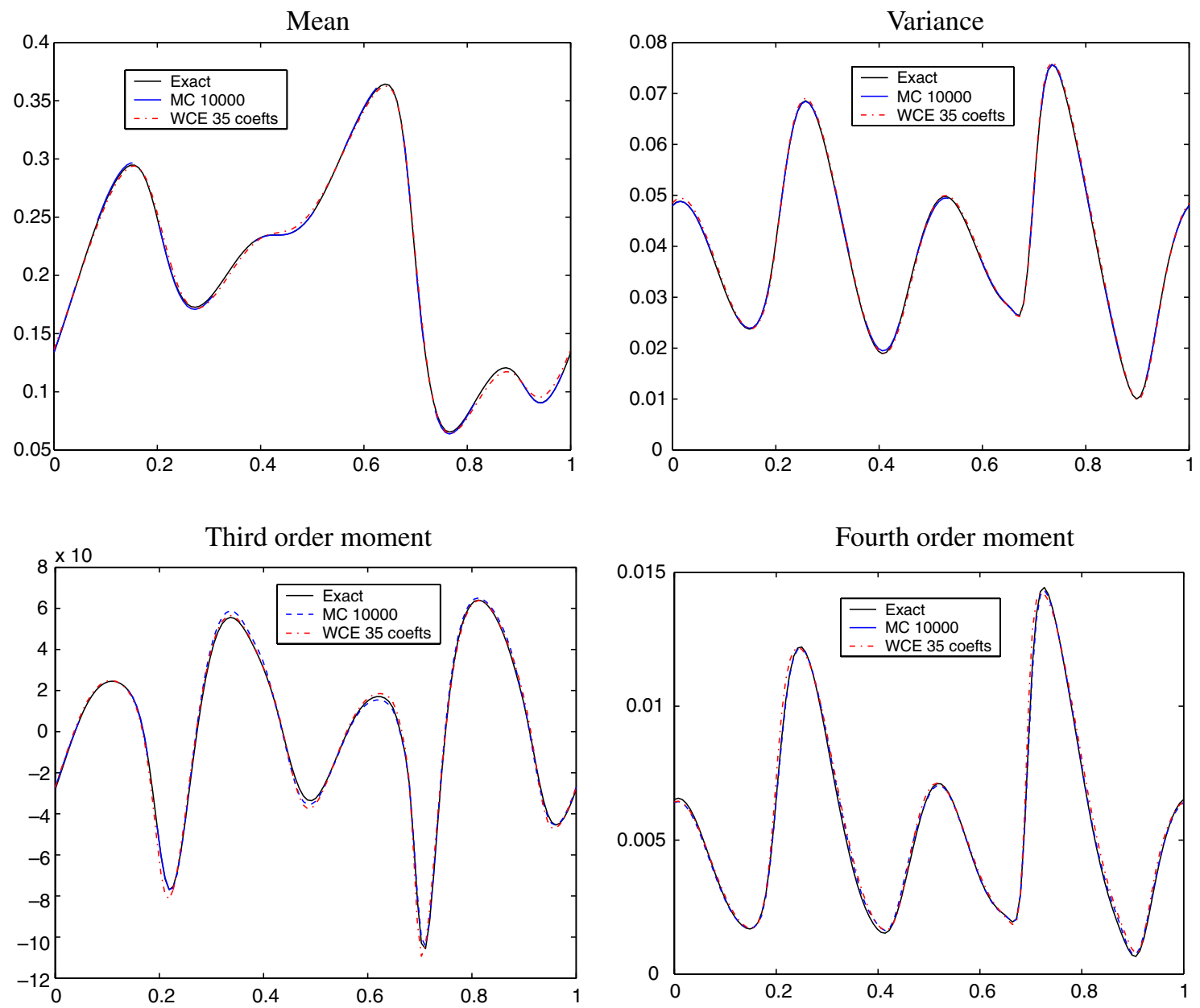

Fig. 3. Convergence comparison between the WCE method and MC simulation. WCE with 35 coefficients has accuracy to that of the accuracy as MC simulation with 10,000 realizations. However, the CPU time is only $2 \mathrm{~s}$ for the WCE method, as opposed to $315 \mathrm{~s}$ for MC. The WCE method is more efficient than MC simulation in this case.

Theorem 3.1 can be easily verified by Itô's formula. The decomposition (3.11) provides a convenient way to compute the statistical moments of $u$ numerically. Indeed, denote $Y(t)=\sigma W(t)$ and $Z(t)=\sigma \int_{0}^{t} W(s) \mathrm{d} s$. It can be shown that the joint probability density function of random variables $(Y, Z)$ is given by $\rho(y, z)=$ $\frac{\sqrt{3}}{\pi \sigma^{2} t^{2}} \exp \left(-\frac{2 y^{2}}{\sigma^{2} t}+\frac{6 y z}{\sigma^{2} t^{2}}-\frac{6 z^{2}}{\sigma^{2} t^{3}}\right)$. Then from (3.11), we have

$$
E[u(x, t)]^{n}=\int_{\mathbf{R}^{2}}[v(x-z, t)+y]^{n} \rho(y, z) \mathrm{d} y \mathrm{~d} z .
$$

To compute the statistical moments of $u$, we can first solve the deterministic Burgers equation (3.12) and obtain the solution $v$, then compute the integrals (3.13) numerically. Since all the computations are deterministic, we can quantify and hence control the numerical error at each step. By carefully choosing the deterministic algorithms, we can compute the statistical moments of $u$ very accurately.

In the following numerical test, we consider the Burgers equation (3.1) with the same setup as in Section 3.2. The only difference is that the spatial part of the random forcing is chosen as a constant $\sigma=0.1$. We first solve the problem to $T=0.8$ by the semi-analytical approach and obtain the benchmark statistics of $u$. Then we solve the same problem by the WCE method. We project the Brownian motion in $[0, T]$ onto the first four Haar wavelet bases, and truncate the WCE expansion at fourth-order Wick polynomials. The resulting 
Table 1

Relative $L^{2}$ error of the WCE method with 40 coefficients and MC simulation with 10,000 realizations

\begin{tabular}{lcllll}
\hline & CPU $(\mathrm{s})$ & Mean & Variance $(\%)$ & Third-order $(\%)$ & Fourth-order $(\%)$ \\
\hline WCE & 3 & 0.08 & 1.3 & 4.3 & 5.2 \\
MC & 319 & 0.18 & 2.1 & 3.8 & 4.1 \\
\hline
\end{tabular}

WCE propagator has 40 coefficients. According to Theorem 5.1, the maximum mean square error for such a truncation is $\approx \mathrm{O}\left(10^{-2}\right)$. For comparison, we also solve the problem by MC simulation with 10,000 realizations. In all the three methods, we use the same spatial mesh $N=128$ and time step $\Delta t=0.001$. We compare the numerical solutions of the WCE method and MC method in Table 1. In the table, the relative $L^{2}$ error for the WCE Mean is defined as $\left\|E\left(u^{\mathrm{WCE}}\right)-E(u)\right\|_{2} /\|E(u)\|_{2}$, where $u$ is the semi-analytical solution. The errors for the other moments are defined similarly. To reach comparable accuracy, the WCE method takes only $3 \mathrm{~s}$, while MC simulation takes $319 \mathrm{~s}$. So the WCE method is much faster than MC simulation for that 1D stochastic Burgers equation. Furthermore, we indeed observed an accuracy of $\mathrm{O}\left(10^{-2}\right)$ for the WCE method as predicted by Theorem 5.1 .

It is worth pointing out that the performance of the WCE method is not sensitive to the choice of $L^{2}$ basis functions, $m_{k}(t)$, as long as they are orthonormal. For example, when we tried both Haar wavelets and the trigonometric basis; the numerical results were almost identical.

\section{WCE method for Navier-Stokes equations}

In this section, we apply the WCE method to solve a 2D stochastic Navier-Stokes equation (SNS). We consider a temperature distribution convected by a stochastic velocity field $\vec{u}$, where $\vec{u}=(u, v)$ is governed by the Navier-Stokes equation driven by Brownian motions:

$$
\left\{\begin{array}{l}
\theta_{t}+\vec{u} \cdot \nabla \theta=\mu \Delta \theta, \\
\vec{u}_{t}+\vec{u} \cdot \nabla \vec{u}=v \Delta \vec{u}-\nabla P+\sigma \dot{\vec{W}}(t), \\
\nabla \cdot \vec{u}=0,
\end{array}\right.
$$

where $\theta$ is the scaled temperature, and $\vec{W}(t)=\left(W_{1}, W_{2}\right)^{\mathrm{T}}$ is a Brownian motion vector with independent components. The matrix $\sigma(x, y)=\operatorname{diag}\left(\sigma_{1}, \sigma_{2}\right)$ accounts for the spatial dependence of the random forcing. $\mu$ and $v$ are the temperature diffusivity and fluid viscosity, respectively. The temperature $\theta$ is convected by the random flow passively, and serves as a visualization media for the fluid particle path. For simplicity, we do not consider the coupling between the temperature and momentum equation.

In our numerical experiment, we take the computational domain as $[0,1]^{2}$, and assume that $\theta$ and $\vec{u}$ are doubly periodic in the domain. We consider the following initial condition for vorticity $\omega=v_{x}-u_{y}$ :

$$
\omega(x, y, 0)=C-\frac{1}{2 \delta} \exp \left(-\frac{I(x)(y-0.5)^{2}}{2 \delta^{2}}\right),
$$

where $I(x)=1+\epsilon(\cos (4 \pi x)-1)$, and $C$ is a constant to make the initial vorticity mean zero: $\int_{[0,1]^{2}} \omega(x, y, 0) \mathrm{d} x \mathrm{~d} y=0$. The initial vorticity is concentrated in a narrow layer centered at $y=0.5$. It describes a flat shear layer of characteristic width $\delta$. However, the width is perturbed sinusoidally with amplitude $\epsilon$. If $\delta$ goes to zero, the initial vorticity approaches a perturbed flat vortex sheet.

The initial condition for temperature is chosen to be

$$
\theta(x, y, 0)= \begin{cases}H_{\delta}(y-0.25), & y \leqslant 0.4 \\ 1-2 H_{\delta}(y-0.5), & 0.4<y<0.6 \\ -H_{\delta}(0.75-y), & y \geqslant 0.6\end{cases}
$$

where $H_{\delta}(x)$ is the mollified Heaviside function 


$$
H_{\delta}(x)= \begin{cases}0 & \text { if } x<-\delta \\ \frac{x+\delta}{2 \delta}+\frac{\sin (\pi x / \delta)}{2 \pi} & \text { if }|x| \leqslant \delta \\ 1 & \text { if } x>\delta\end{cases}
$$

The initial temperature is comprised of four smoothly connected layers. The interfaces between the different layers have the same thickness $\delta$ as the initial vorticity.

In this paper, we choose $\delta=0.025$, which gives rise to a sharp shear layer and temperature interfaces. The perturbation to the initial vortex layer is set to $\epsilon=0.3$. The initial vorticity and temperature are plotted in Fig. 4. Due to the Kelvin-Helmholtz instability, the fluid will roll up and convect the temperature layers. The roll-up of a thin shear layer is a well-known challenging problem in computational fluid mechanics [20]. With the extra complexity of Brownian motion, the stochastic problem (4.1) provides a severe test for the WCE method.

\subsection{The WCE method}

In this subsection, we discuss how to solve the stochastic Navier-Stokes equation (4.1) by the WCE method. In the two-dimensional case, the stochastic Navier-Stokes equation can be rewritten in the stream functionvorticity formulation. Define the vorticity variable $\omega=v_{x}-u_{y}$, and introduce the stream function $\psi$ such that $\vec{u}=\left(\psi_{y},-\psi_{x}\right)$, we can rewrite the original system (4.1) as

$$
\left\{\begin{array}{l}
\theta_{t}+(u \theta)_{x}+(v \theta)_{y}=\mu \Delta \theta, \\
\omega_{t}+(u \omega)_{x}+(v \omega)_{y}=v \Delta \omega+\left(\sigma_{2}\right)_{x} \dot{W}_{2}-\left(\sigma_{1}\right)_{y} \dot{W}_{1}, \\
-\Delta \psi=\omega, \\
u=\psi_{y}, \quad v=-\psi_{x} .
\end{array}\right.
$$

We assume that the stream function $\psi$ is also doubly periodic in the domain $[0,1]^{2}$.

We proceed exactly as we did for the $1 \mathrm{D}$ stochastic Burgers equation. We choose an arbitrary orthonormal basis $m_{i}(t), i=1,2, \ldots$, in $L^{2}([0, T])$, where $[0, T]$ is the time interval in which we want to solve the problem. Then we project each Brownian motion component $\left\{W_{k}(t), 0 \leqslant t \leqslant T\right\}$ to the $L^{2}$ basis:

$$
\xi_{i}^{k}=\int_{0}^{T} m_{i}(t) \mathrm{d} W_{k}(t), \quad i=1,2, \ldots, \quad k=1,2 .
$$
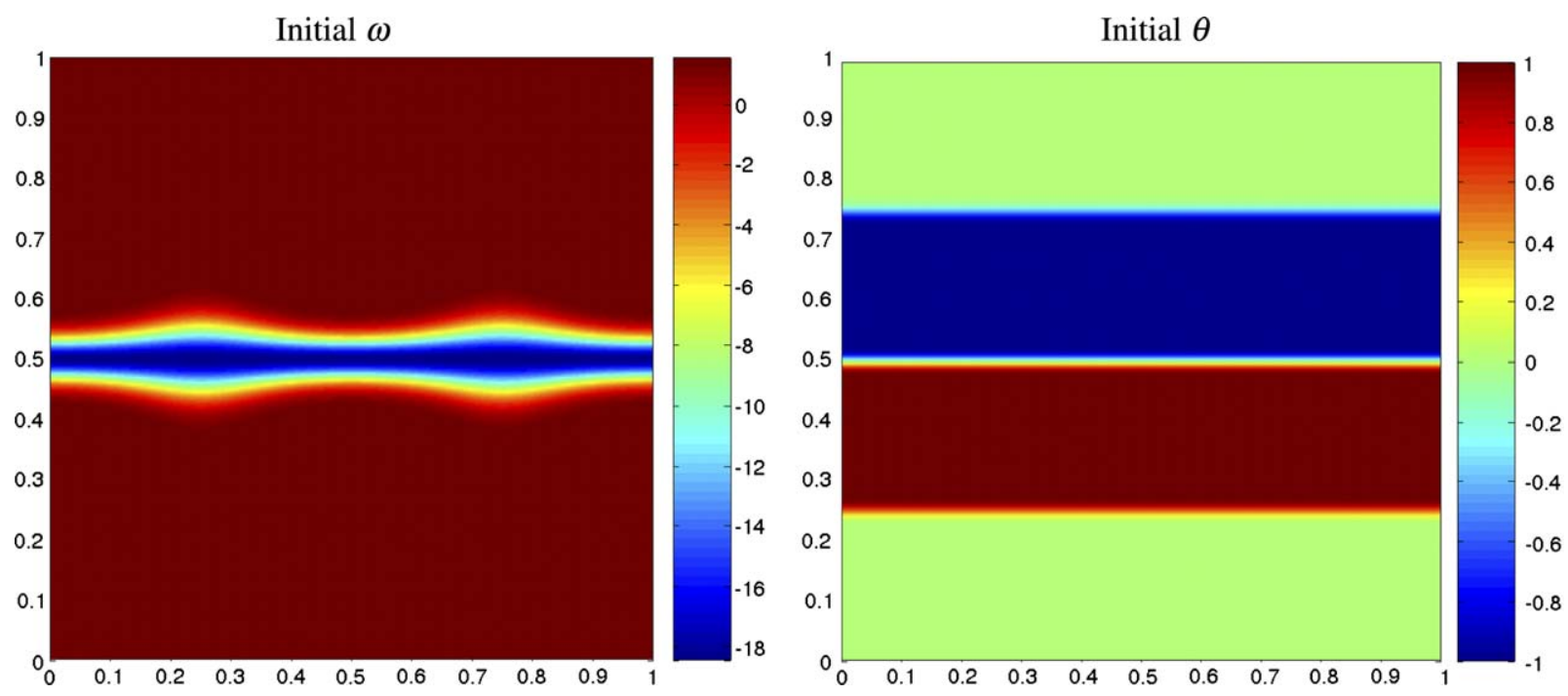

Fig. 4. Initial vorticity $\omega$ and temperature $\theta$. 
Let $\alpha=\left(\alpha_{i}^{k}, i=1,2, \ldots, k=1,2\right)$ be a multi-index with non-negative integer components. Denote the finite multi-index set as $\mathscr{J}=\left\{\alpha,|\alpha|=\sum_{i, k}\left|\alpha_{i}^{k}\right|<\infty\right\}$. For $\alpha \in \mathscr{J}$, define the Wick polynomial $T_{\alpha}=$ $\left.\prod_{k=1}^{2} \prod_{i=1}^{\infty} H_{\alpha_{i}^{k}} \xi_{i}^{k}\right)$. Suppose $\theta, \omega, \vec{u}$, and $\psi$ admit the Wiener Chaos expansions and $\theta_{\alpha}, \omega_{\alpha}, \vec{u}_{\alpha}$, and $\psi_{\alpha}$ are their WCE coefficients. Similar to the 1D stochastic Burgers equation, we can derive the WCE propagator for system (4.5):

$$
\left\{\begin{array}{l}
\left(\theta_{\alpha}\right)_{t}+\mathscr{L}_{\alpha}(u, \theta)_{x}+\mathscr{L}_{\alpha}(v, \theta)_{y}=\mu \Delta \theta_{\alpha}, \\
\left(\omega_{\alpha}\right)_{t}+\mathscr{L}_{\alpha}(u, \omega)_{x}+\mathscr{L}_{\alpha}(v, \omega)_{y}=v \Delta \omega_{\alpha}+\left(\sigma_{2}\right)_{x} I_{\{|\alpha|=1\}} \sum_{j=0}^{\infty} \alpha_{j}^{2} m_{j}(t)-\left(\sigma_{1}\right)_{y} I_{\{|\alpha|=1\}} \sum_{i=0}^{\infty} \alpha_{i}^{1} m_{i}(t), \\
-\Delta \psi_{\alpha}=\omega_{\alpha}, \quad u_{\alpha}=\left(\psi_{\alpha}\right)_{y}, \quad v_{\alpha}=-\left(\psi_{\alpha}\right)_{x} .
\end{array}\right.
$$

In the above formula, the bilinear operator $\mathscr{L}_{\alpha}(\cdot, \cdot)$ is defined as

$$
\mathscr{L}_{\alpha}(f, g)=\sum_{p \in \mathscr{J}} \sum_{0 \leqslant \beta \leqslant \alpha} C(\alpha, \beta, p) f_{\beta+p} g_{\alpha-\beta+p}
$$

for $f=\sum_{\alpha \in \mathscr{F}} f_{\alpha} T_{\alpha}$ and $g=\sum_{\beta \in \mathscr{f}} g_{\beta} T_{\beta}$. The coefficient $C(\alpha, \beta, p)$ is defined in (3.3). The indicator function $I_{\{|\alpha|=1\}}=1$ if $|\alpha|=1$ and it is zero otherwise.

In the following numerical experiment, we set $\mu=0.0002, v=0.0002$. In the stream function-vorticity formulation (4.5), the spatial parts of the random forcing are taken as

$$
\left(\sigma_{1}\right)_{y}=0.1 \pi \cos (2 \pi x) \cos (2 \pi y), \quad\left(\sigma_{2}\right)_{x}=0.1 \pi \cos (2 \pi x) \sin (2 \pi y) .
$$

We solve the problem to $T=1.0$ by the WCE method. We choose to project each Brownian motion component onto the first four Haar wavelet bases, and truncate the WCE approximation at fourth-order Wick polynomials. The resulting system should have 495 coefficients. However, using the sparse tensor product approach laid out in Section 3.2, the total number of coefficients can be reduced to 56. We discretize the computational domain by a $256 \times 256$ square mesh, and choose $\Delta t=0.00125$. Then we solve the truncated WCE propagator on the domain $[0,1]^{2}$ by Fourier pseudo-spectral method. The CPU time is $8.1 \mathrm{~h}$. The mean and variance of $\theta$ and $\omega$ are plotted in Fig. 5. Due to the Kelvin-Helmholtz instability, the mean vorticity rolls up and forms a pair of concentrated vortex eyes. The temperature layers are convected and diffused by the random velocity. The sharp edges of the interface are smeared, due to both the diffusivity and the random forcing. The variances of the vorticity and temperature all concentrate along the sharp layers of the interface and is almost zero at the constant regions. In this sense, the random forcing acts as an extra diffusion for the mean equation, even though a closed form of the mean equation is not available.

Another observation is that the third-order moment of both the vorticity and temperature are not zero. Actually, they have the same magnitude and similar structure as the respective variances. This shows that the solution of the stochastic Navier-Stokes equation is far from being a Gaussian process.

To reach the similar accuracy as the WCE method, MC simulation needs thousands of realizations, which will take more than two days to compute. For this 2D stochastic Navier-Stokes equation, it is clear that the WCE method is more efficient than the MC simulation for short time integrations. Since it is not easy to obtain a benchmark solutions by MC simulation or any other method, we will demonstrate the convergence of the WCE method for a special case in the following subsection.

\subsection{Convergence verification of the WCE method}

To test the convergence of the WCE method for stochastic Navier-Stokes equations, we consider a special case of Eq. (4.1), where a semi-analytical solution is available. Similar to 1D Burgers equation, we have the following result.

Theorem 4.1. In system (4.1), if the spatial part $\sigma(x, y)$ of the random forcing is a constant matrix, then the stochastic solutions of (4.1) can be expressed as 
$\omega$ mean

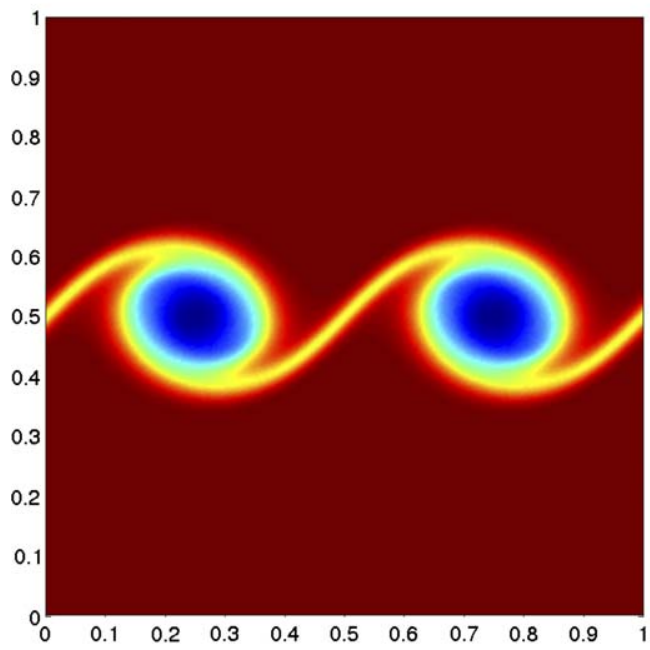

$\omega$ variance

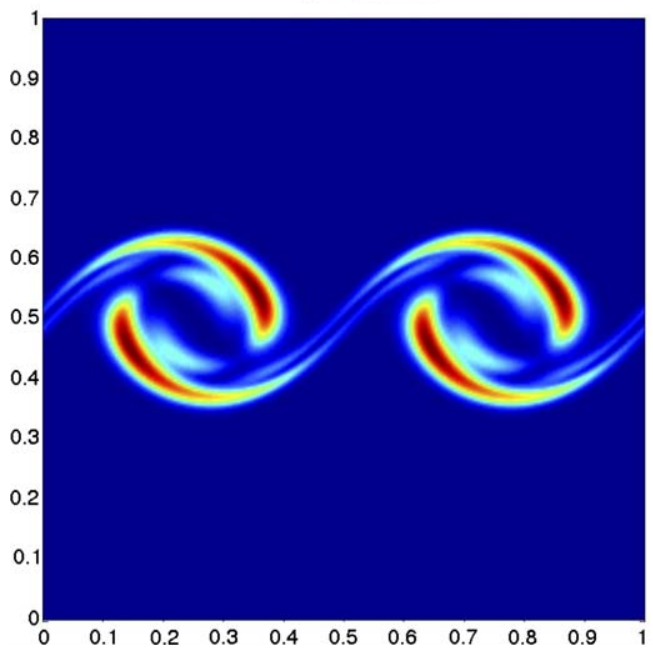

$\theta$ mean

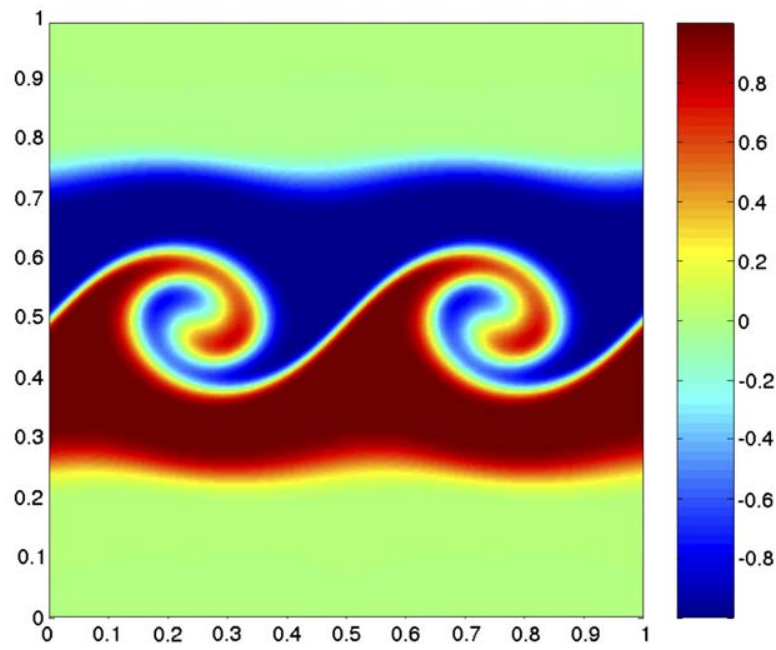

$\theta$ variance

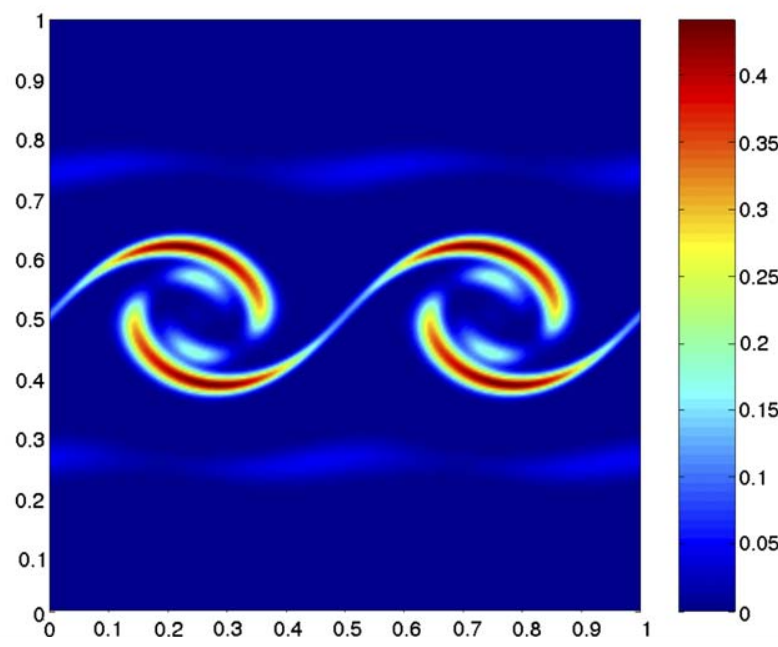

Fig. 5. Mean and variance of vorticity $\omega$ and temperature $\theta$ at $T=1.0$, computed by the WCE method with 56 coefficients.

$$
\begin{aligned}
& \theta(\vec{x}, t)=\theta^{\prime}\left(\vec{x}-\int_{0}^{t} \sigma \vec{W}(s) \mathrm{d} s, t\right), \quad P(\vec{x}, t)=P^{\prime}\left(\vec{x}-\int_{0}^{t} \sigma \vec{W}(s) \mathrm{d} s, t\right), \\
& \vec{u}(\vec{x}, t)=\vec{u}^{\prime}\left(\vec{x}-\int_{0}^{t} \sigma \vec{W}(s) \mathrm{d} s, t\right)+\sigma \vec{W}(t),
\end{aligned}
$$

where $\theta^{\prime}, \vec{u}^{\prime}, P^{\prime}$ are solutions of the corresponding unforced deterministic equations

$$
\left\{\begin{array}{l}
\theta_{t}^{\prime}+\vec{u}^{\prime} \cdot \nabla \theta^{\prime}=\mu \Delta \theta^{\prime}, \\
\vec{u}_{t}^{\prime}+\vec{u}^{\prime} \cdot \nabla \vec{u}^{\prime}=v \Delta \vec{u}^{\prime}-\nabla P^{\prime}, \quad \nabla \cdot \vec{u}^{\prime}=0 .
\end{array}\right.
$$

Theorem 4.1 is readily verified by Itô's formula. For the random vorticity $\omega$, we can further obtain

$$
\omega(\vec{x}, t)=\omega^{\prime}\left(\vec{x}-\int_{0}^{t} \sigma \vec{W}(s) \mathrm{d} s, t\right),
$$

where $\omega^{\prime}=v_{x}^{\prime}-u_{y}^{\prime}$ is the deterministic vorticity of the unforced problem (4.10). 
Table 2

Relative $L_{2}$ error on the domain $[0,1]^{2}$ of the WCE method with 56 coefficients and the MC simulation with 1000 realizations

\begin{tabular}{llllll}
\hline & CPU $(\mathrm{h})$ & $E(\omega)(\%)$ & $E(\theta)(\%)$ & $\operatorname{Var}(\omega)(\%)$ & $\operatorname{Var}(\theta)(\%)$ \\
\hline WCE & 9.3 & 0.028 & 0.044 & 1.2 & 1.7 \\
MC & 44.7 & 0.23 & 0.36 & 8.2 & 8.8 \\
\hline
\end{tabular}

The semi-analytical forms (4.9) and (4.11) state that the stochastic solutions are the deterministic solutions of the corresponding unforced problem compounded with a random perturbation along the flow characteristics. That observation can help us better understand the effects of the random forcing. Formula (4.9) and (4.11) also provide a convenient way to compute the statistical moments of $\theta$ and $\omega$ numerically. Indeed, for any fixed $x, t$ and integer $n=1,2, \ldots$, it is easy to show that

$$
\begin{aligned}
& E[\theta(\vec{x}, t)]^{n}=\int_{\mathbf{R}^{2}}\left[\theta^{\prime}(\vec{x}-\vec{z}, t)\right]^{n} \rho(\vec{z}, t) \mathrm{d} \vec{z}, \\
& E[\omega(\vec{x}, t)]^{n}=\int_{\mathbf{R}^{2}}\left[\omega^{\prime}(\vec{x}-\vec{z}, t)\right]^{n} \rho(\vec{z}, t) \mathrm{d} \vec{z},
\end{aligned}
$$

where

$$
\rho(\vec{z}, t)=\frac{3}{2 \pi \sigma_{1} \sigma_{2} t^{3}} \exp \left(-\frac{3 z_{1}^{2}}{2 \sigma_{1}^{2} t^{3}}-\frac{3 z_{2}^{2}}{2 \sigma_{2}^{2} t^{3}}\right) .
$$

To obtain the benchmark statistics of $\theta$ and $\omega$, we can first solve the deterministic Eq. (4.10), then compute the statistical moments from (4.12) and (4.13) by numerical integrations. Under this framework, all computations are deterministic and no random number generation is needed. By carefully choosing the deterministic algorithms, we can compute the benchmark statistics of $\theta$ and $\omega$ very accurately.

In the next numerical experiment, we set the temperature diffusivity as $\mu=0.0005$, and the fluid viscosity as $v=0.0005$. The constants in the random forcing are chosen as $\sigma_{1}=0.02, \sigma_{2}=0.02$. Since the random forcing is spatially independent, its effect will not be damped out in time by viscosity. To avoid excessive smearing induced by the random forcing, we deliberately choose $\sigma_{1}, \sigma_{2}$ not much larger than the physical viscosity $v$.

We first solve the problem to $T=1.0$ by the semi-analytical method and obtain the benchmark statistics. To test the performance of the WCE method and the MC simulation, we apply both of them to the same problem and compare their numerical solutions with that of the semi-analytical approach. For the WCE method, we project each Brownian motion component onto the first four wavelet bases, and truncate the WCE propagator at fourth-order Wick polynomials. Altogether 56 coefficients are retained in the computation. The maximum mean square error for such a truncation is $\approx \mathrm{O}\left(10^{-2}\right)$, based on the error analysis similar to that in Theorem 5.1. We also solve the problem to $T=1.0$ by MC simulation with 1000 realizations. For a fair comparison, the same spatial mesh $256 \times 256$ and time step $\Delta t=0.00125$ are used for all three different methods. The numerical solutions are compared in Table 2. The WCE method with 56 coefficients is more accurate than the MC simulation with 1000 realizations for all the statistical moments computed. In addition, the WCE method is also several times faster than the MC simulation. To reach the same accuracy as the WCE method, the MC simulation may need much more than 1000 realizations, which will take even more CPU time. However, the WCE method requires more computer memory than the MC simulation since a coupled PDE system for the WCE coefficients has to be solved simultaneously. For the 1D and 2D problems considered in this paper, the memory usage is not a big issue and all the computation can be done on a standard PC. For 3D stochastic Navier-Stokes equations, we expect that the memory usage will become a bigger concern to the WCE method when many WCE coefficients have to be retained.

\section{Error analysis of the WCE method}

Due to the nonlinear coupling in the WCE propagator, it is very difficult to conduct an error analysis of the truncated WCE method in the general setting. In this section, we only establish an error bound for the WCE approximation when the random forcing is independent of the spatial variables. In this case the solutions of 
the stochastic Navier-Stokes and Burgers equations could be obtained from the respective deterministic counterparts by a stochastic Galilean transformation. Though a special case, the error bound in this particular setting sheds light on the general performance of the WCE method. For simplicity, we will prove the result in the context of the stochastic Burgers equation. For the stochastic Navier-Stokes equation, the proof and results are very similar.

Consider the stochastic Burgers equation

$$
u_{t}+\frac{1}{2}\left(u^{2}\right)_{x}=\mu u_{x x}+\sigma \dot{W}(t), \quad u(x, 0)=u_{0}(x),
$$

where $\sigma$ is a constant. According to Theorem 3.1, the solution of (5.1) can be expressed as

$$
u(x, t)=v\left(x-\sigma \int_{0}^{t} W(s) \mathrm{d} s, t\right)+\sigma W(t)
$$

where $v(x, t)$ is the solution of the unforced deterministic Burgers equation

$$
v_{t}+\frac{1}{2}\left(v^{2}\right)_{x}=\mu v_{x x}, \quad v(x, \mathbf{0})=u_{0}(x)
$$

Suppose the basis functions $m_{k}(s)$ in the space $L^{2}([0, t])$ are chosen as follows: $m_{1}(s)=\frac{1}{\sqrt{t}}, m_{k}(s)=$ $\sqrt{\frac{2}{t}} \cos \left(\frac{(k-1) \pi s}{t}\right), 0 \leqslant s \leqslant t, k \geqslant 2$. Write $\mathscr{J}_{K, N}=\left\{\alpha, \alpha=\left(\alpha_{1}, \ldots, \alpha_{K}\right),|\alpha| \leqslant N\right\}$. The main result of this section is as follows:

Theorem 5.1. Let

$$
u_{K, N}(x, t)=\sum_{\alpha \in \mathscr{F}_{K, N}} u_{\alpha}(x, t) T_{\alpha}, \quad \text { where } \quad u_{\alpha}(x, t)=E\left[u(x, t) T_{\alpha}\right] .
$$

Then, the error estimate

$$
\max _{x} \sqrt{E\left|u(x, t)-u_{K, N}(x, t)\right|^{2}} \leqslant B_{N+1} \frac{\sqrt{(2 N+1) ! !}}{(N+1) !} \sigma^{N+1}\left(\frac{t^{3}}{3}\right)^{\frac{N+1}{2}}+B_{1} \sigma\left(\frac{t}{K}\right)^{3 / 2}
$$

holds, where $B_{n}=\sup _{x}\left|\frac{\partial^{n}}{\partial x^{n}} v(x, t)\right|$ and $v$ is the solution of the deterministic Eq. (5.3). ${ }^{1}$

Proof. Denote $Z(t)=\int_{0}^{t} W(s) \mathrm{d} s$. Owing to (2.3), the Wiener process $W(s), s \in[0, t]$, admits the following expansion:

$$
W(s)=\frac{s}{\sqrt{t}} \xi_{1}+\sum_{k=2}^{\infty} \xi_{k} \frac{\sqrt{2 t}}{(k-1) \pi} \sin \left(\frac{(k-1) \pi s}{t}\right) .
$$

Hence, $Z=Z_{K}+Z_{R}$, where

$$
\begin{aligned}
& Z_{K}=\frac{\sqrt{t^{3}}}{2} \xi_{1}+\sum_{k=2}^{K} C_{k} \frac{\sqrt{t^{3}}}{(k-1)^{2}} \xi_{k}, \\
& Z_{R}=\sum_{k=K+1}^{\infty} C_{k} \frac{\sqrt{t^{3}}}{(k-1)^{2}} \xi_{k},
\end{aligned}
$$

where $C_{k}=\frac{\sqrt{2}}{\pi^{2}}\left[1+(-1)^{k}\right]$. Obviously $Z_{K}$ and $Z_{R}$ are orthogonal in that $E\left(Z_{K} Z_{R}\right)=0$.

Note that $W(t)=\sqrt{t} \xi_{1}$ and the solution (5.2) can be rewritten as

$$
u(x, t)=v\left(x-\sigma Z_{K}-\sigma Z_{R}, t\right)+\sigma \sqrt{t} \xi_{1} .
$$

Expanding $v\left(x-\sigma Z_{K}-\sigma Z_{R}, t\right)$ in Taylor's series, firstly with respect to $Z_{R}$ and then with respect to $Z_{K}$, we arrive at

\footnotetext{
${ }^{1}$ A similar but more general error estimate for WCE method in Linear SPDEs can be found in [19].
} 


$$
u(x, t)=v\left(x-\sigma Z_{K}, t\right)+I_{1}+\sigma \sqrt{t} \xi_{1}=v(x, t)+\sum_{n=1}^{N} \frac{\left(-\sigma Z_{K}\right)^{n}}{n !} \frac{\partial^{n} v}{\partial x^{n}}(x, t)+I_{2}+I_{1}+\sigma \sqrt{t} \xi_{1},
$$

where

$$
I_{1}(x, t)=-\frac{\partial v}{\partial x}\left(x-\sigma Z_{K}-\theta_{1}, t\right) \sigma Z_{R}, I_{2}(x, t)=\frac{\left(-\sigma Z_{K}\right)^{N+1}}{(N+1) !} \frac{\partial^{N+1} v}{\partial x^{N+1}}\left(x-\theta_{2}, t\right)
$$

are the Lagrange residuals of the Taylor expansions.

Denote

$$
\tilde{u}_{K, N}(x, t)=v(x, t)+\sigma \sqrt{t} \xi_{1}+\sum_{n=1}^{N} \frac{\left(-\sigma Z_{K}\right)^{n}}{n !} \frac{\partial^{n} v}{\partial x^{n}}(x, t) .
$$

Obviously, $\tilde{u}_{K, N}(x, t)$ is a polynomial of $\xi_{1}, \ldots, \xi_{K}$ with maximum order $N$. Since the WCE truncation $u_{K, N}(x, t)$ is a Hermite polynomial expansion, which is an orthogonal projection with respect to the Gaussian measure, it follows that

$$
\sqrt{E\left|u(x, t)-u_{K, N}(x, t)\right|^{2}} \leqslant \sqrt{E\left|u(x, t)-\tilde{u}_{K, N}(x, t)\right|^{2}} \leqslant B_{1} \sigma \sqrt{E\left|Z_{R}^{2}\right|}+B_{N+1} \frac{\sigma^{N+1}}{(N+1) !} \sqrt{E\left|Z_{K}^{2 N+2}\right|},
$$

where $B_{n}=\sup _{x}\left|\frac{\partial^{n}}{\partial x^{n}} v(x, t)\right|$. From formula (5.7) we have

$$
E\left|Z_{R}^{2}\right| \leqslant \frac{8 t^{3}}{\pi^{4}} \sum_{k=K+1}^{\infty} \frac{1}{(k-1)^{4}}<\frac{8 t^{3}}{\pi^{4}}\left(\frac{1}{K^{4}}+\int_{K}^{\infty} \frac{\mathrm{d} x}{x^{4}}\right)<\frac{t^{3}}{K^{3}} .
$$

Note that $Z_{K}$ is a linear combination of centered Gaussian random variables, so it is a centered Gaussian itself. Due to properties of Gaussian random variables, we have

$$
E\left|Z_{K}^{2 N+2}\right|=\left(E\left|Z_{K}^{2}\right|\right)^{N+1}(2 N+1) ! !<\left(E\left|Z^{2}\right|\right)^{N+1}(2 N+1) ! !
$$

It is easy to show that $E\left|Z^{2}\right|=t^{3} / 3$. Substituting (5.11) and (5.12) into (5.10), we obtain

$$
\sqrt{E\left|u(x, t)-u_{K, N}(x, t)\right|^{2}} \leqslant B_{N+1} \frac{\sqrt{(2 N+1) ! !}}{(N+1) !} \sigma^{N+1}\left(\frac{t^{3}}{3}\right)^{\frac{N+1}{2}}+B_{1} \sigma \sqrt{\frac{t^{3}}{K^{3}}} .
$$

Since the right-hand side does not depend on $x$, taking the maximum in $x$ recovers the estimate (5.5). This completes the proof.

Remark. The first term in the error estimate (5.5) reflects the error in truncating the Fourier-Hermite expansion of the solution. Making use of the Stirling formula, one can easily check that the asymptotic of this term is given by

$$
\frac{\sqrt{(2 N+1) ! !}}{(N+1) !} \sigma^{N+1}\left(\frac{t^{3}}{3}\right)^{\frac{N+1}{2}} \sim C\left(\frac{2 \sigma^{2} t^{3} e / 3}{N+1}\right)^{\frac{N+1}{2}} .
$$

This confirms our numerical observation that the WCE approximation converges exponentially in terms of the Wick polynomial order $N$.

The second term in (5.5) stems from the truncation error of the Fourier expansion (5.6), which is given by

$$
E\left[\int_{0}^{t}\left|W(s)-\sum_{k=1}^{K} \xi_{k} \int_{0}^{s} m_{k}(\tau) \mathrm{d} \tau\right|^{2} \mathrm{~d} s\right]<\frac{C}{K} .
$$

The slow convergence of the second term is a reflection of the irregularity of the Brownian motion forcing.

Both Theorem 5.1 and the numerical experiments presented above indicate that the convergence rate of the WCE method critically depends on the time span $[0, t]$, the magnitude of the random forcing $\sigma$, and the regularity of the solution. Clearly, the smaller the time interval $[0, t]$ for the Wiener Chaos expansion, the faster 
the convergence. Note that Brownian motion has independent increments. When long time simulations are of interest, one can divide a large time interval into small ones and apply the WCE method repeatedly on those small intervals. If the time subinterval is of size $\Delta t$, then by Theorem 5.1 the error of approximation for each step is $\mathrm{O}\left((\Delta t)^{3 / 2}\right)$. Hence, the global rate of convergence should be $\mathrm{O}(\sqrt{\Delta t})$.

A smaller forcing would increase the rate of convergence of the WCE method. In a broad class of SPDEs arising in physics and engineering, the large structures and dominant dynamic effects are captured by the deterministic physical laws, while the unresolved small scales, microscopic effects, and other uncertainties are often modeled as stochastic perturbations with small amplitudes. We expect that the WCE method will prove to be especially effective in applications to this class of problems.

Based on Theorem 5.1, the rate of convergence of WCE method also depends on $\sup _{x}\left|\frac{\partial^{n}}{\partial x^{n}} v(x, t)\right|$ where $v$ is a solution of an appropriate deterministic equation of fluid dynamics. Conditions that guarantee boundedness of $\left|\frac{\partial^{n}}{\partial x^{n}} v(x, t)\right|$ are well known. For example, if $v_{0} \in W_{p}^{n}\left(R^{2}\right)$ for some $p>2$, then for any $T<\infty$ $\sup _{x, t} \frac{\partial^{n}}{\partial x^{n}} v(x, t) \mid<\infty$ (see, e.g. [14]). Also, this provides an indication that at large gradient regions (rough flow regions), more WCE terms are needed to obtain the same accuracy.

\section{Remarks on long time integration and variability}

As it was demonstrated in the previous sections, as long as the time interval is comparatively short, e.g. $T<1.0$, the performance of the WCE is quite satisfactory. However, long time integrations of the WCE method present a substantial challenge. For long time integrations, one has to solve the equation step-by-step on successive small time intervals. In Section 5, it was shown that the truncated WCE expansion would still converge even for large $T$ at the rate $\mathrm{O}(\sqrt{\Delta t})$, where $\Delta t$ is the size of the time step. Yet, when the number of time steps grows, so does the number of the Wick polynomials $T_{\alpha}$. Moreover, the later grows at a much higher rate. To remedy this problem, one has to find a ways to keep the number of the basis functions $T_{\alpha}$ involved to be reasonably small at every time step. This is a work in progress, so we only briefly outline a promising approach here.

Consider the Euler time discretization of the Burgers equation (5.1):

$$
u^{n+1}=u^{n}+\left(\mu u_{x x}^{n}-u_{x}^{n} u^{n}\right) \Delta t+\sigma \sqrt{\Delta t} \xi_{n+1},
$$

where $\xi_{n+1}:=(W((n+1) \Delta t)-W(n \Delta t)) / \sqrt{\Delta t} \sim \mathscr{N}(0,1)$. The WCE coefficients $u_{\alpha}^{n+1}$ of $(6.1)$ are computed by solving the propagator equation

$$
u_{\alpha}^{n+1}=I_{\left(\alpha_{n+1}=0\right)}\left(u_{\alpha}^{n}+\left(v \Delta u_{\alpha}^{n}-\sum_{p \in \mathscr{J}_{n}} \sum_{0 \leqslant \beta \leqslant \alpha} C(\alpha, \beta, p) \frac{\partial}{\partial x}\left(u_{p+\alpha-\beta}^{n} u_{p+\beta}^{n}\right)\right) \Delta t\right)+I_{\left(\alpha_{n+1}=1, \alpha \mid=1\right)} \sigma \sqrt{\Delta t},
$$

where $\mathscr{J}_{n}:=\left\{\alpha: \alpha_{i}=0\right.$ for $\left.i>n\right\}$. Clearly, $u^{n+1}$ can be expressed as a linear combination of $\xi_{n+1}$ and Wick polynomials of $\left(\xi_{i}, i \leqslant n\right)$ with order up to $2^{n}$. However, if a random variable $\xi_{i}$ is presented in a Wick polynomial by its $k$ th power, it is also multiplied by $(\sigma \sqrt{\Delta t})^{k}$. Thus if $\sigma \sqrt{\Delta t} \ll 1$, the growth of the number of Wick polynomials is automatically discounted by the rapid decrease of its weight. This effect opens up a possibility for effective aggregation and truncation of the step-by-step WCE expansions. We have carried out some numerical experiments using a simplest version of this strategy. The preliminary results are encouraging. However, more systematic study along this line of research is required. This will be reported in detail elsewhere. The adaptive truncation strategy described above is similar in spirit to those proposed by Ghanem, etc. [18] and Karniadakis, etc. [21]. However, their method is not readily applicable to stochastic PDEs with Brownian motion forcing, which are what we considered in this paper.

The performance of the WCE method not only depends on the length of the time interval $[0, T]$ but also on the magnitude of the random forcing. Indeed, the $L^{2}$ norm of the random forcing $E\left[\int_{0}^{T} \sigma \mathrm{d} W(t)\right]^{2}=\int_{0}^{T} \sigma^{2} \mathrm{~d} t$ measures the random effect that we need to resolve. Larger $T$ or $\sigma$ mean stronger random input. As a result, more WCE bases are required to resolve the random dependence of the stochastic solution. In this sense, the difficulty with large random forcing is equivalent to that of long time integration.

Next, we test how the magnitude of the random forcing affects the performance of the WCE method. In the stochastic Burgers equation (3.1), we set the spatial part of the random forcing as 

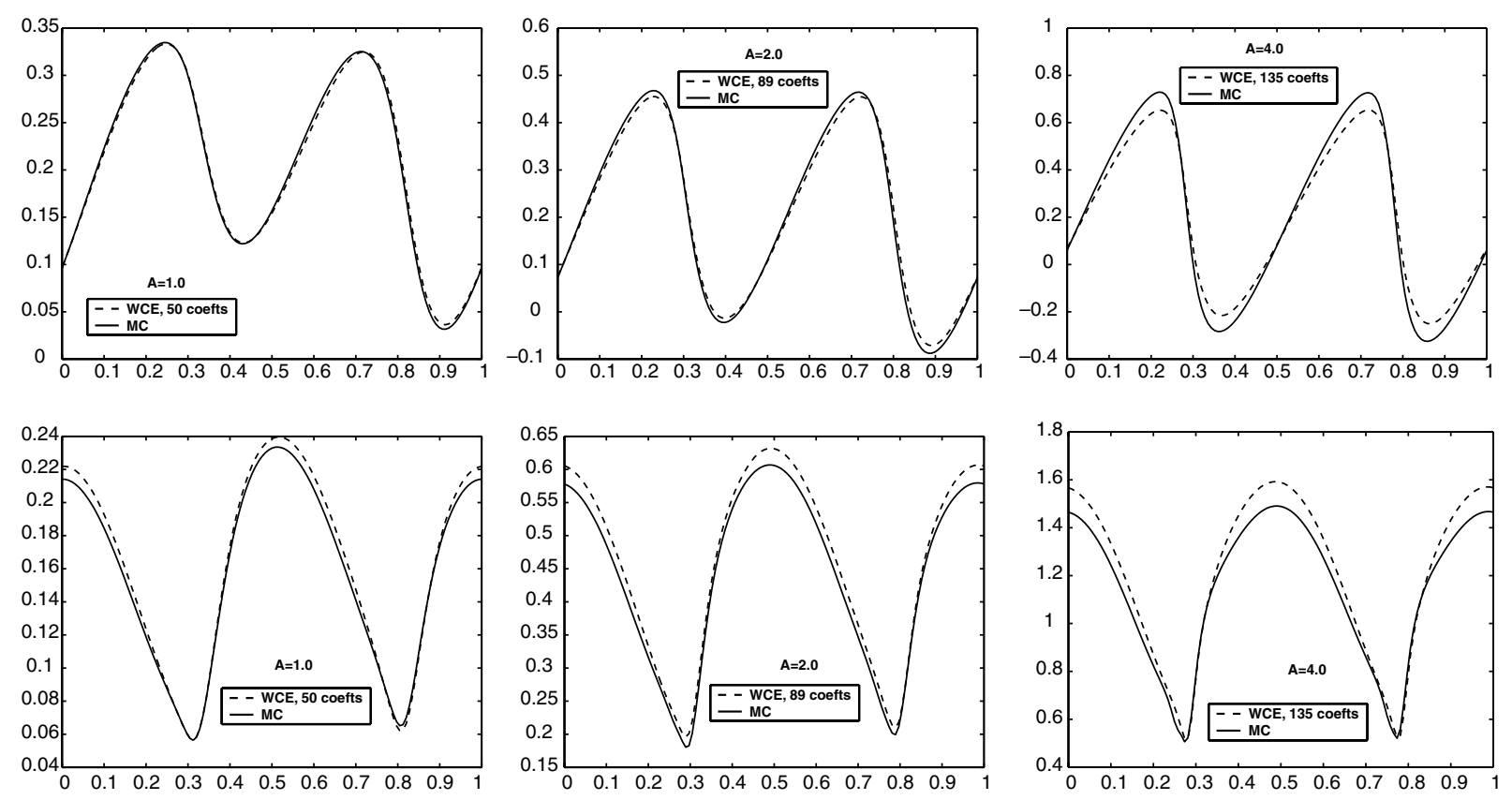

Fig. 6. Mean (first row) and variance (second row) for different magnitudes of forcing. The first column: $A=1.0$; the second column: $A=2.0$; the third column: $A=4.0$. In all three cases, 10,000 realizations are computed in MC simulation. When $A$ increases from 1.0 to 4.0, we need more and more WCE bases to achieve similar accuracy. However, the WCE method can approximate the right solution reasonably well when enough coefficients are retained.

$$
\sigma(x)=A \cos (2 \pi x)
$$

where $A$ is a constant. The viscosity is chosen as $\mu=0.02$. We solve the problem to $T=0.8$ for $A=1.0,2.0$, 4.0 , respectively. The numerical results are plotted in Fig. 6 . When the magnitude of the random forcing increases, we need to include more and more WCE bases to achieve similar accuracy. Theoretically, the increase of the magnitude of the forcing does not lead to a blowup. Our numerical experiments, while limited, also indicate that the WCE method is quite stable and does not blow up even when hundreds of the coefficients are included.

\section{Conclusion and discussion}

In this paper, we have explored a spectral numerical method for solving equations of fluid dynamics (FD) driven by Brownian motion. The approach is based on Wiener Chaos expansion methodology. Loosely speaking, WCE could be viewed as a Fourier series with respect to the driving white noise (rather than the spatial or temporal variables). It turns out that there exists a closed infinite system of deterministic equations for the related Fourier coefficients. This system, which we call the "propagator", describes how an equation of fluid dynamics (e.g. a Navier-Stokes equation) propagates chaos that is generated by the driving Brownian motion. The propagator is a deterministic system of PDEs with the nonlinearity structure that is typical of FD equations. Hence, with minimal modifications, the existing methods developed in CFD can be applied to solving the propagator.

Numerical examples were provided for stochastic Burgers equations with periodic boundary conditions and a 2D stochastic Navier-Stokes equation coupled with transport equation. The WCE method provides simple and rigorous analytical formulae for computing statistical moments. In our simulations the statistical moments up to the fourth order were computed.

In the numerical experiments with stochastic Burgers and Navier-Stokes equations, we have compared the solution accuracy and the computational effectiveness of the WCE and the Monte Carlo (MC) approaches. It appears that at the same level of accuracy, and especially when the resolution level is high, the computational 
cost of the MC method is notably higher. Another advantage of the WCE method as compared to the MC simulation is that the former does not require the generation of random numbers.

The analytical error analysis of the WCE method was carried out and the bounds of errors resulting from truncation of the WCE expansion were established. It was shown that the local error of estimate is proportional to $(\Delta t)^{3 / 2}$ where $\Delta t$ is the time step.

There is, however, a number of unresolved issues. In particular, further research is required on:

1. Long time computations. In this paper, we mainly discuss the short time computations. Since the local error estimate is proportional to $(\Delta t)^{3 / 2}$, the global rate of convergence should be $\mathrm{O}(\Delta t)^{1 / 2}$. The practicality of this conclusion, however, is subject to efficient control of the number of sources of randomness involved. The number of random variables (generated by increments of the driving Brownian motion) grows in time. This results in fast growth of the number of stochastic basis $\left\{T_{\alpha}\right\}$. In order to make the numerical scheme effective, we need to be able to compress the "history" without losing too much information. This problem is being addressed in our ongoing research.

2. Error analysis. In this paper is limited to the case of random forcing independent of spatial variables. It appears that the error analysis methodology developed in [19] could be expanded to the general setting of this paper but this task is beyond the scope of this paper.

3. The dimensionality. All the simulations in this paper are limited to one- and two-dimensional equations. The theoretical base of the WCE method is independent of the spatial dimensions; However, the numerical implementation is obviously dimension sensitive. Further work on this subject is in order.

\section{Acknowledgements}

This paper was supported in part by NSF Grant DMS-0073916 and DMS-0410062, NSF ITR Grant ACI0204932, ONR Grant N00014-03-1-0823, and ARO Grant DAAD19-02-1-0374. The authors thank Prof. Russell Caflisch for helpful discussion, Roger Donaldson and Jessica Printz for proofreading the paper, and the referees for revision suggestions.

\section{References}

[1] J. Bec, U. Frisch, K. Khanin, Kicked Burgers turbulence, J. Fluid Mech. 418 (2000) 239-267.

[2] A. Bensoussan, R. Temam, Equations stochastiques du type Navier-Stokes, J. Func. Anal. 13 (1973) $195-222$.

[3] R.H. Cameron, W.T. Martin, The orthogonal development of non-linear functionals in series of Fourier-Hermite functionals, Ann. Math. 48 (1947) 385-392.

[4] G. Da Prato, A. Debussche, R. Temam, Stochastic Burgers' equation, Nonlinear Differ. Eq. Appl. 1 (4) (1994) $389-402$.

[5] G. Da Prato, A. Debussche, Ergodicity for the 3D stochastic Navier-Stokes equations, J. Math. Pure Appl. 82 (2003) $877-947$.

[6] A.J. Chorin, Hermite expansion in Monte-Carlo simulations, J. Comput. Phys. 8 (1971) 472-482.

[7] A.J. Chorin, Gaussian fields and random flow, J. Fluid Mech. 63 (1974) 21-32.

[8] R. Courant, D. HilbertMethods of Mathematical Physics, vol. 1, Wiley-Interscience, New York, 1953.

[9] S.C. Crow, G.H. Canavan, Relationship between a Wiener-Hermite expansion and an energy cascade, J. Fluid Mech. 41 (1970) 387.

[10] P. Frauenfelder, C. Schwab, R.A. Todor, Finite elements for elliptic problems with stochastic coefficients, Comput. Methods Appl. Mech. Eng. 194 (2005) 205-228.

[11] R.G. Ghanem, P.D. Spanos, Stochastic Finite Elements: A Spectral Approach, Springer, Berlin, 1991.

[12] T. Hida, H.H. Kuo, J. Potthoff, L. Streit, White Noise, Kluwer Academic Publishers, Dordrecht, 1993.

[13] M. Jardak, C.H. Su, G.E. Karniadakis, Spectral polynomial chaos solutions of the stochastic advection equation, J. Sci. Comput. 17 (2002) 319-338.

[14] T. Kato, G. Ponce, Well-Posedness of the Euler and Navier-Stokes equations in the Lebesgue Spaces $L_{s}^{p}\left(R^{2}\right)$, Rev. Mat. Iberoamericana 2 (1-2) (1986) 73-88.

[15] W.E.K. Khanin, A. Mazel, Ya. Sinai, Probability distribution functions for the random forced Burgers equation, Phys. Rev. Lett. 78 (1997) 1904-1907.

[16] W.E.K. Khanin, A. Mazel, Ya. Sinai, Invariant measures for Burgers equation with stochastic forcing, Ann. Math. 151 (2000) $877-$ 960.

[17] R.H. Kraichnan, Small-scale structure of a scalar field convected by turbulence, Phys. Fluids 11 (1968) 487-489.

[18] R. Li, R. Ghanem, Adaptive polynomial chaos expansions applied to statistics of extremes in nonlinear random vibration, Prob. Eng. Mech. 13 (1998) 125-136. 
[19] S. Lototsky, R. Mikulevicius, B. Rozovskii, Nonlinear filtering revisited: a special approach, SIAM J. Control Optim. 35 (1997) 435461.

[20] J.S. Lowengrub, M.J. Shelley, B. Merriman, High-order and efficient methods for the vorticity formulation of the Euler equations, SIAM J. Sci. Comput. 14 (1993) 1107-1142.

[21] D. Lucor, G.E. Karniadakis, Adaptive generalized polynomial chaos for nonlinear random oscillators, SIAM J. Sci. Comput. 26 (2) (2004) 720-735.

[22] R. Mikulevicius, B.L. Rozovskii, Stochastic Navier-Stokes equations. Propagation of chaos and statistical moments, in: J.L. Menaldi, E. Rofmann, A. Sulem (Eds.), Optimal Control and Partial Differential Equations. In honor of Professor Alain Bensoussan's 60th Birthday, IOS Press, Amsterdam, 2001, pp. 258-267.

[23] R. Mikulevicius, B. Rozovskii, Stochastic Navier-Stokes equations for turbulence flow, SIAM J. Math. Anal. 35 (2004) 1250-1310.

[24] S.A. Orszag, L.R. Bissonnette, Dynamical properties of truncated Wiener-Hermite expansions, Phys. Fluids 10 (1967) 2603.

[25] G. Papanicolaou, Wave propagation in a one-dimensional random medium, SIAM J. Appl. Math. 21 (1971) $13-18$.

[26] G. Papanicolaou, Diffusion in random media, in: J.B. Keller, D. McLaughlin, G. Papanicolaou (Eds.), Surveys in Applied Mathematics, Plenum Press, New York, 1995, pp. 205-255.

[27] S. Sakamoto, R. Ghanem, Polynomial chaos decomposition for the simulation of non-Gaussian non-stationary stochastic processes, J. Eng. Mech. 128 (2002) 190-201.

[29] D. Zhang, Z. Lu, An efficient, high-order perturbation approach for flow in random porous media via Karhunen-Loève and polynomial expansion, J. Comput. Phys. 194 (2004) 773-794.

[30] D. Xiu, G.E. Karniadakis, Modeling uncertainty in flow simulations via generalized polynomial chaos, J. Comput. Phys. 187 (2003) 137-167.

[31] D. Xiu, G.E. Karniadakis, The Wiener-Askey polynomial chaos for stochastic differential equations, SIAM J. Sci. Comput 24 (2002) 619-644. 